\title{
Fourth generation effects in processes induced by $b \rightarrow s$ transition
}

\author{
T. M. Aliev ${ }^{a *}$, A. Özpineci ${ }^{b \dagger}$, M. Savci ${ }^{a} \ddagger$ \\ a Physics Department, Middle East Technical University, 06531 Ankara, Turkey \\ b The Abdus Salam International Center for Theoretical Physics, I-34100, Trieste, Italy
}

\begin{abstract}
We study the effects of sequential fourth quark generation in rare $B \rightarrow K\left(K^{*}\right) \ell^{+} \ell^{-}$ decays induced by $b \rightarrow s$ transition and in $B_{s}^{0}-\bar{B}_{s}^{0}$ mixing. Using the experimental values on branching ratios of the $B \rightarrow X_{s} \gamma$ and $B \rightarrow K\left(K^{*}\right) \ell^{+} \ell^{-}$decays, the allowed regions for $\left|V_{t b} V_{t s}^{*}\right|$ and $\left|V_{t^{\prime} b} V_{t^{\prime} s}^{*}\right|$ are determined as a function of the $t^{\prime}$ quark mass.
\end{abstract}

PACS numbers: 12.60.-i, 13.20.--v, 13.20.He

*e-mail: taliev@metu.edu.tr

${ }^{\dagger}$ e-mail: ozpineci@ictp.trieste.it

‡e-mail: savci@metu.edu.tr 


\section{Introduction}

Despite Standard Model (SM) describes successfully all low energy experiments, it is an incomplete theory. This theory contains many unsolved and fundamental problems, such as the origin of $\mathrm{CP}$ violation, mass spectrum and the number of generations. Recent observation of neutrino oscillations [1] indicated that neutrino sector of SM must be enlarged. One of the most straightforward and economical extension of the SM is adding the fourth generation to the fermionic sector, similar to the three-generation case. The extra generation can contribute to the electroweak radiative corrections. Latest studies in the electroweak sector allow the existence of a fourth generation with heavy Dirac neutrino $[2,3]$. Moreover, two or three extra generations with relatively "light" neutrinos, with a mass about $50 \mathrm{GeV}$ are also allowed [3]. Flavor-changing neutral current (FCNC) transitions provide potentially the most sensitive and stringiest test for the SM at loop level, since they are forbidden in the $\mathrm{SM}$ at tree level. At the same time these transitions are very sensitive to the new physics beyond the SM via contributions of the new particles to the loop diagrams. It should be stressed that if newly proposed particles are heavy and if they cannot be produced directly in the accelerators, their influence through the loop diagrams can be a unique possibility for establishing new physics beyond the SM. The effects of the fourth generation to rare decays have been studied in many works [4]-[8].

Although theoretically FCNC processes are highly suppressed in the SM, very exciting results are obtained on the experimental side. The first measurement of the FCNC processes through $b \rightarrow s \gamma$ were reported by CLEO [9]. Recently, $B \rightarrow K \ell^{+} \ell^{-}$decay is observed at B factories at SLAC and KEK [10]-[12]. BaBar Collaboration also reported their preliminary results about the observation of $B \rightarrow K^{*} \ell^{+} \ell^{-}$decay with branching ratio $\mathcal{B}\left(B \rightarrow K^{*} \ell^{+} \ell^{-}\right)=\left(1.68_{-0.58}^{+0.68} \pm 0.28\right) \times 10^{-6}$ and $90 \%$ C.L. $\mathcal{B}\left(B \rightarrow K^{*} \ell^{+} \ell^{-}\right)<3 \times 10^{-6}$ [12]. In this paper, we study the contributions of the fourth generation to the processes induced by the $b \rightarrow s$ transitions and use the experimental results of the branching ratio for the $b \rightarrow s \gamma[13], B \rightarrow K \ell^{+} \ell^{-}$and $B \rightarrow K^{*} \ell^{+} \ell^{-}$decays and try to determine the constraints on the extended Cabibbo-Kobayashi-Maskawa matrix (CKM) elements $\left|V_{t b} V_{t s}^{*}\right|$ and $\left|V_{t^{\prime} b} V_{t^{\prime} s}^{*}\right|$.

The paper is organized as follows. In section 2, we present the basic theoretical expressions for the differential widths of the $B \rightarrow K \ell^{+} \ell^{-}$and $B \rightarrow K^{*} \ell^{+} \ell^{-}$decays, and for the mass difference $\Delta m_{B_{s}}$, with sequential up-like quark in the fourth generation model. Section 3 is devoted to the numerical analysis and the conclusion.

\section{Theoretical results}

In this section we present the necessary theoretical formulae for the $B \rightarrow X_{s} \gamma, B \rightarrow K \ell^{+} \ell^{-}$, $B \rightarrow K^{*} \ell^{+} \ell^{-}$decays and for the mass difference in the $B_{s}^{0}-\bar{B}_{s}^{0}$ system in presence of the fourth generation. All these processes, except mixing in the $B_{s}^{0}-\bar{B}_{s}^{0}$ system, are induced by the $b \rightarrow s$ transition. At quark level this transition is described by the effective Hamiltonian

$$
\mathcal{H}_{\text {eff }}=\frac{\alpha G_{F}}{2 \sqrt{2} \pi} V_{t b} V_{t s}^{*} \sum_{i=1}^{10} C_{i}(\mu) \mathcal{O}_{i}(\mu)
$$


where the full set of operators in $\mathcal{O}_{i}(\mu)$ and the corresponding expressions for the Wilson coefficients in the SM3 (here and in all further discussions SM3 and SM4 will denote SM with three and four generations, respectively) are given in $[14,15]$. As is well known, the fourth generation introduces the first three generations into SM copiously, and hence it is clear that it changes only values of the Wilson coefficients $C_{7}(\mu), C_{9}(\mu)$ and $C_{10}(\mu)$ with the help of the running fourth generation up quark $t^{\prime}$ at loop level, and do not introduce any new operator structure, i.e.,

$$
\begin{aligned}
& C_{7}^{t o t}(\mu)=C_{7}^{S M}(\mu)+\frac{V_{t^{\prime} b} V_{t^{\prime} s}^{*}}{V_{t b} V_{t s}^{*}} C_{7}^{t^{\prime}}(\mu), \\
& C_{9}^{t o t}(\mu)=C_{9}^{S M}(\mu)+\frac{V_{t^{\prime} b} V_{t^{\prime} s}^{*}}{V_{t b} V_{t s}^{*}} C_{9}^{t^{\prime}}(\mu), \\
& C_{10}^{t o t}(\mu)=C_{10}^{S M}(\mu)+\frac{V_{t^{\prime} b} V_{t^{\prime} s}^{*}}{V_{t b} V_{t s}^{*}} C_{10}^{t^{\prime}}(\mu),
\end{aligned}
$$

where $V_{t^{\prime} b}$ and $V_{t^{\prime} s}$ are the elements of the $4 \times 4$ Cabibbo-Kobayashi-Maskawa (CKM) matrix. The explicit forms of the $C_{i}^{t^{\prime}}$ can easily be obtained from the SM results by simply substituting $m_{t} \rightarrow m_{t^{\prime}}$. Neglecting the $s$ quark mass, the effective Hamiltonian leads to the following matrix element for the $b \rightarrow s \ell^{+} \ell^{-}$transition

$$
\begin{aligned}
\mathcal{M} & =\frac{G \alpha}{2 \sqrt{2} \pi} V_{t b} V_{t s}^{*}\left[C_{9}^{t o t} \bar{s} \gamma_{\mu}\left(1-\gamma_{5}\right) b \bar{\ell} \gamma_{\mu} \ell+C_{10}^{t o t} \bar{s} \gamma_{\mu}\left(1-\gamma_{5}\right) b \bar{\ell} \gamma_{\mu} \gamma_{5} \ell\right. \\
& \left.-2 C_{7}^{t o t} \frac{m_{b}}{q^{2}} \bar{s} \sigma_{\mu \nu} q^{\nu}\left(1+\gamma_{5}\right) b \bar{\ell} \gamma_{\mu} \ell\right]
\end{aligned}
$$

where $q^{2}=\left(p_{1}+p_{2}\right)^{2}$ and $p_{1}$ and $p_{2}$ are the four-momenta of the final leptons. We observe from Eq. (3) that in order to calculate the matrix element for the $B \rightarrow K^{*}(K) \ell^{+} \ell^{-}$decay, the matrix elements of the quark operators in Eq. (3) need to be sandwiched between initial and final $\left(K\right.$ or $\left.K^{*}\right)$ meson states, which results in a form that is parametrized in terms of the form factors

$$
\begin{aligned}
& \left\langle K^{*}\left(p_{K^{*}}, \varepsilon\right)\left|\bar{s} \gamma_{\mu}\left(1-\gamma_{5}\right) b\right| B\left(p_{B}\right)\right\rangle= \\
& \quad-i \varepsilon_{\mu}^{*}\left(m_{B}+m_{K^{*}}\right) A_{1}\left(q^{2}\right)+i\left(p_{B}+p_{K^{*}}\right)_{\mu}\left(\varepsilon^{*} q\right) \frac{A_{2}\left(q^{2}\right)}{m_{B}+m_{K^{*}}} \\
& \quad+i q_{\mu} \frac{2 m_{K^{*}}}{q^{2}}\left(\varepsilon^{*} q\right)\left[A_{3}\left(q^{2}\right)-A_{0}\left(q^{2}\right)\right]-\epsilon_{\mu \nu \lambda \sigma} \varepsilon^{* \nu} p_{K^{*}}^{\lambda} q^{\sigma} \frac{2 V\left(q^{2}\right)}{m_{B}+m_{K^{*}}}
\end{aligned}
$$

where $\varepsilon$ is the polarization vector of $K^{*}$ meson and $q=p_{B}-p_{K^{*}}$ is the momentum transfer. Using the equation of motion, the form factor $A_{3}\left(q^{2}\right)$ can be written in terms of $A_{1}\left(q^{2}\right)$ and $A_{2}\left(q^{2}\right)$ as follows

$$
A_{3}\left(q^{2}\right)=\frac{\left(m_{B}+m_{K^{*}}\right)}{2 m_{K^{*}}} A_{1}\left(q^{2}\right)-\frac{\left(m_{B}-m_{K^{*}}\right)}{2 m_{K^{*}}} A_{2}\left(q^{2}\right)
$$

In order to ensure that there exists no kinematical singularity we assume that $A_{3}\left(q^{2}=0\right)=$ $A_{0}\left(q^{2}=0\right)$. 
The corresponding form factors are defined through the matrix elements for the $B \rightarrow K$ transition as

$$
\left\langle K\left(p_{K}\right)\left|\bar{s} \gamma_{\mu} b\right| B\left(p_{B}\right)\right\rangle=f_{+}\left[\left(p_{B}+p_{K}\right)_{\mu}-\frac{m_{B}^{2}-m_{K}^{2}}{q^{2}} q_{\mu}\right]+f_{0} \frac{m_{B}^{2}-m_{K}^{2}}{q^{2}} q_{\mu} .
$$

Finetness of Eq. (6) is guaranteed by demanding $f_{+}(0)=f_{0}(0)$.

The semileptonic form factors for the $K^{*}$ and $K$ mesons resulting from the dipole operator $\bar{s} i \sigma_{\mu \nu} q^{\nu}\left(1+\gamma_{5}\right) b$ are defined as

$$
\begin{gathered}
\left\langle K^{*}\left(p_{K^{*}}, \varepsilon\right)\left|\bar{s} i \sigma_{\mu \nu} q^{\nu}\left(1+\gamma_{5}\right) b\right| B\left(p_{B}\right)\right\rangle= \\
2 \epsilon_{\mu \nu \lambda \sigma} \varepsilon^{* \nu} p_{K^{*}}^{\lambda} q^{\sigma} T_{1}\left(q^{2}\right)+i\left[\varepsilon_{\mu}^{*}\left(m_{B}^{2}-m_{K^{*}}^{2}\right)-\left(p_{B}+p_{K^{*}}\right)_{\mu}\left(\varepsilon^{*} q\right)\right] T_{2}\left(q^{2}\right) \\
+i\left(\varepsilon^{*} q\right)\left[q_{\mu}-\left(p_{B}+p_{K^{*}}\right)_{\mu} \frac{q^{2}}{m_{B}^{2}-m_{K^{*}}^{2}}\right] T_{3}\left(q^{2}\right), \\
\left\langle K\left(p_{K}\right)\left|\bar{s} i \sigma_{\mu \nu} q^{\nu}\left(1+\gamma_{5}\right) b\right| B\left(p_{B}\right)\right\rangle=-\frac{f_{T}}{m_{B}+m_{K}}\left[\left(p_{B}+p_{K}\right)_{\mu} q^{2}-q_{\mu}\left(m_{B}^{2}-m_{K}^{2}\right)\right] .
\end{gathered}
$$

The matrix elements of the $B \rightarrow K \ell^{+} \ell^{-}$and $B \rightarrow K^{*} \ell^{+} \ell^{-}$decays can be written as

$$
\mathcal{M}=\frac{G \alpha}{2 \sqrt{2} \pi} V_{t b} V_{t s}^{*} m_{B}\left(F_{\mu}^{1 i} \bar{\ell} \gamma_{\mu} \ell+F_{\mu}^{2 i} \bar{\ell} \gamma_{\mu} \gamma_{5} \ell\right)
$$

where $i=1$ corresponds to $K$ meson and $i=2$ corresponds to $K^{*}$ meson, respectively, and

$$
\begin{aligned}
& F_{\mu}^{11}=\frac{1}{m_{B}}\left[A^{\prime}\left(p_{B}+p_{K}\right)_{\mu}+B^{\prime} q_{\mu}\right] \\
& F_{\mu}^{21}=\frac{1}{m_{B}}\left[C^{\prime}\left(p_{B}+p_{K}\right)_{\mu}+D^{\prime} q_{\mu}\right] \\
& F_{\mu}^{12}=\left[-\frac{1}{m_{B}^{2}} A \epsilon_{\mu \nu \rho \sigma} \varepsilon^{* \nu} p_{K^{*}}^{\rho} q^{\sigma}-i B_{1} \varepsilon_{\mu}^{*}+i \frac{1}{m_{B}^{2}} B_{2}\left(\varepsilon^{*} q\right) p_{K^{*} \mu}+i \frac{1}{m_{B}^{2}} B_{3}\left(\varepsilon^{*} q\right) q_{\mu}\right], \\
& F_{\mu}^{22}=\left[-\frac{1}{m_{B}^{2}} C_{1} \epsilon_{\mu \nu \rho \sigma} \varepsilon^{* \nu} p_{K^{*}}^{\rho} q^{\sigma}-i D_{1} \varepsilon_{\mu}^{*}+i \frac{1}{m_{B}^{2}} D_{2}\left(\varepsilon^{*} q\right) p_{K^{*} \mu}+i \frac{1}{m_{B}^{2}} D_{3}\left(\varepsilon^{*} q\right) q_{\mu}\right],
\end{aligned}
$$

where

$$
\begin{aligned}
A^{\prime} & =C_{9}^{t o t} f_{+}+2 \frac{m_{b}}{m_{B}} \frac{1}{(1+\sqrt{r})} C_{7}^{t o t} f_{T}, \\
B^{\prime} & =C_{9}^{t o t} f_{+}+2 \frac{m_{b}}{m_{B}} \frac{(1-\sqrt{r})}{s} C_{7}^{t o t} f_{T}, \\
C^{\prime} & =C_{10}^{t o t} f_{+}, \\
D^{\prime} & =C_{10}^{t o t} f_{-}, \\
A & =\frac{2 V}{1+\sqrt{r}} C_{9}^{t o t}+4 \frac{m_{b}}{m_{B} s} C_{7}^{t o t} T_{1}, \\
B_{1} & =(1+\sqrt{r})\left[C_{9}^{t o t} A_{1}+2 \frac{m_{b}}{m_{B} s}(1-\sqrt{r}) C_{7}^{t o t} T_{2}\right],
\end{aligned}
$$




$$
\begin{aligned}
B_{2} & =\frac{1}{1-r}\left[(1-\sqrt{r}) C_{9}^{t o t} A_{2}+2 \frac{m_{b}}{m_{B}} C_{7}^{t o t}\left(T_{3}+\frac{1-r}{s} T_{2}\right)\right], \\
B_{3} & =\frac{1}{s}\left[2 \sqrt{r} C_{9}^{e f f}\left(A_{3}-A_{0}\right)-2 \frac{m_{b}}{m_{B}} C_{7}^{t o t} T_{3}\right], \\
C_{1} & =\frac{2 V}{1+\sqrt{r}} C_{10}^{t o t}, \\
D_{1} & =(1+\sqrt{r}) C_{10}^{t o t} A_{1}, \\
D_{2} & =\frac{A_{2}}{1+\sqrt{r}} C_{10}^{t o t}, \\
D_{3} & =\frac{2 \sqrt{r}}{s}\left(A_{3}-A_{0}\right) C_{10}^{t o t},
\end{aligned}
$$

where

$$
f_{-}\left(q^{2}\right)=\frac{(1-r)}{s}\left[f_{0}\left(q^{2}\right)-f_{+}\left(q^{2}\right)\right], \quad r=\frac{m_{K^{*}}^{2}}{m_{B}^{2}}, \quad s=\frac{q^{2}}{m_{B}^{2}} .
$$

Using the matrix element, for the dilepton invariant mass distribution we get

$$
\begin{aligned}
\frac{d \Gamma^{B \rightarrow K}}{d s} & =\frac{G^{2} \alpha^{2} m_{B}^{5}}{2^{10} \pi^{5}}\left|V_{t b} V_{t s}^{*}\right|^{2} \sqrt{\lambda} v\left\{\frac{\lambda}{3}\left(3-v^{2}\right)\left(\left|A^{\prime}\right|^{2}+\left|C^{\prime}\right|^{2}\right)+s\left(1-v^{2}\right)(2+2 r-s)\left|C^{\prime}\right|^{2}\right. \\
& \left.+2 s\left(1-v^{2}\right)(1-r) \operatorname{Re}\left[C^{\prime} D^{\prime *}\right]+s^{2}\left(1-v^{2}\right)\left|D^{\prime}\right|^{2}\right\} \\
\frac{d \Gamma^{B \rightarrow K^{*}}}{d s} & =\frac{G^{2} \alpha^{2} m_{B}^{5}}{2^{10} \pi^{5}}\left|V_{t b} V_{t s}^{*}\right|^{2} \sqrt{\lambda} v\left\{\frac{1}{6} s \lambda\left(3-v^{2}\right)|A|^{2}+\frac{1}{3} s \lambda v^{2}\left|C_{1}\right|^{2}\right. \\
& +\frac{1}{12 r}\left[\left(3-v^{2}\right)(\lambda+12 r s)\left|B_{1}\right|^{2}+\left(\lambda\left(3-v^{2}\right)+24 r s v^{2}\right)\left|D_{1}\right|^{2}\right] \\
& +\frac{\lambda}{12 r}\left[\lambda\left(3-v^{2}\right)\left|B_{2}\right|^{2}+\left\{\lambda\left(3-v^{2}\right)+3\left(1-v^{2}\right) s(2+2 r-s)\right\}\left|D_{2}\right|^{2}\right) \\
& -\frac{\lambda}{6 r}\left[\left(3-v^{2}\right)(1-r-s) \operatorname{Re}\left[B_{1} B_{2}^{*}\right]+\left\{\left(3-v^{2}\right)(1-r-s)+3\left(1-v^{2}\right) s\right\} \operatorname{Re}\left[D_{1} D_{2}^{*}\right]\right] \\
& \left.-\frac{\lambda}{2 r}\left(1-v^{2}\right) s\left(\operatorname{Re}\left[D_{1} D_{3}^{*}\right]-(1-r) \operatorname{Re}\left[D_{2} D_{3}^{*}\right]\right)+\frac{\lambda}{4 r}\left(1-v^{2}\right) s^{2}\left|D_{3}\right|^{2}\right\}
\end{aligned}
$$

where $v^{2}=1-4 m_{\ell}^{2} / q^{2}$ and $\lambda(a, b, c)=a^{2}+b^{2}+c^{2}-2 a b-2 a c-2 b c$ is the usual triangle function.

In constraining up quark type fourth generation effects, we will also consider $B_{s}^{0}-\bar{B}_{s}^{0}$ mixing. The mass difference $\Delta m_{B_{s}}$ in SM4 can be written as

$$
\begin{aligned}
\Delta m_{B_{s}} & =\frac{G^{2} m_{W}^{2}}{6 \pi^{2}} m_{B_{s}} B_{B_{s}} f_{B_{s}}^{2}\left\{\eta_{t}\left(V_{t b} V_{t s}^{*}\right)^{2} S_{0}\left(x_{t}\right)+\eta_{t^{\prime}}\left(V_{t^{\prime} b} V_{t^{\prime} s}^{*}\right)^{2} S_{0}\left(x_{t^{\prime}}\right)\right. \\
& \left.+2 \eta_{t t \prime}\left(V_{t b} V_{t s}^{*}\right)\left(V_{t^{\prime} b} V_{t^{\prime} s}^{*}\right) S\left(x_{t}, x_{t^{\prime}}\right)\right\}
\end{aligned}
$$


where $x_{t}=m_{t}^{2} / m_{W}^{2}, x_{t^{\prime}}=m_{t^{\prime}}^{2} / m_{W}^{2}$ and

$$
\begin{aligned}
S_{0}\left(x_{t}\right) & =\frac{4 x_{t}-11 x_{t}^{2}+x_{t}^{3}}{4\left(1-x_{t}\right)^{2}}-\frac{3}{2} \frac{x_{t}^{3} \ln x_{t}}{\left(1-x_{t}\right)^{3}}, \\
S_{0}\left(x_{t^{\prime}}\right) & =S_{0}\left(x_{t} \rightarrow x_{t^{\prime}}\right), \\
S\left(x_{t}, x_{t^{\prime}}\right) & =x_{t} x_{t^{\prime}}\left\{\frac{1}{x_{t^{\prime}}-x_{t}}\left[\frac{1}{4}+\frac{3}{2} \frac{1}{1-x_{t^{\prime}}}-\frac{3}{4} \frac{1}{\left(1-x_{t^{\prime}}\right)^{2}}\right] \ln x_{t^{\prime}}\right. \\
& -\frac{1}{x_{t^{\prime}}-x_{t}}\left[\frac{1}{4}+\frac{3}{2} \frac{1}{1-x_{t}}-\frac{3}{4} \frac{1}{\left(1-x_{t}\right)^{2}}\right] \ln x_{t} \\
& \left.-\frac{3}{4} \frac{1}{\left(1-x_{t}\right)\left(1-x_{t^{\prime}}\right)}\right\} .
\end{aligned}
$$

Here $\eta_{t}=0.55$ is the QCD correction factor. Taking into account the threshold effect from $b^{\prime}$ quark, $\eta_{t t^{\prime}}$ is estimated to be [7]

$$
\eta_{t t^{\prime}}=\left(\alpha_{s}\left(m_{t}\right)\right)^{6 / 23}\left(\frac{\alpha_{s}\left(m_{b^{\prime}}\right)}{\alpha_{s}\left(m_{t}\right)}\right)^{6 / 21}\left(\frac{\alpha_{s}\left(m_{t^{\prime}}\right)}{\alpha_{s}\left(m_{b^{\prime}}\right)}\right)^{6 / 19}
$$

Note that when $m_{t^{\prime}}$ lies between $250 \mathrm{GeV}$ and $400 \mathrm{GeV}, \eta_{t t^{\prime}}$ is quite close to $\eta_{t^{\prime}}$ numerically, hence for simplicity, in further analysis we will set $\eta_{t t^{\prime}}=\eta_{t^{\prime}}$.

In order to obtain quantitative results the value of the fourth generation CKM matrix element $V_{t^{\prime} b} V_{t^{\prime} s}^{*}$ is needed. For this aim we will use the experimentally measured values of the branching ratios $\mathcal{B}\left(B \rightarrow X_{s} \gamma\right)$ and $\mathcal{B}\left(B \rightarrow X_{c} e \bar{\nu}_{e}\right)$. To eliminate the uncertainty coming from $b$ quark mass we consider the ratio

$$
R=\frac{\mathcal{B}\left(B \rightarrow X_{s} \gamma\right)}{\mathcal{B}\left(B \rightarrow X_{c} e \bar{\nu}_{e}\right)}
$$

In leading logarithmic approximation this ratio is equal to

$$
R=\frac{6 \alpha\left|C_{7}^{t o t}\left(m_{b}\right) V_{t b} V_{t s}^{*}\right|^{2}}{\pi f\left(\hat{m}_{c}\right) \kappa\left(\hat{m}_{c}\right)\left|V_{c b}\right|^{2}}
$$

where $\hat{m}_{c}=m_{c} / m_{b}$ and the functions $f\left(\hat{m}_{c}\right)$ and $\kappa\left(\hat{m}_{c}\right)$ for the $b \rightarrow c \ell \bar{\nu}$ transition are given by $[16]$

$$
\begin{aligned}
& f\left(\hat{m}_{c}\right)=1-8 \hat{m}_{c}^{2}+8 \hat{m}_{c}^{6}-\hat{m}_{c}^{8}-24 \hat{m}_{c}^{4} \ln \left(\hat{m}_{c}\right) \\
& \kappa\left(\hat{m}_{c}\right)=1-\frac{2 \alpha_{s}\left(m_{b}\right)}{3 \pi}\left[\left(\pi^{2}-\frac{31}{4}\right)\left(1-\hat{m}_{c}^{2}\right)^{2}+\frac{3}{2}\right] .
\end{aligned}
$$

From Eqs. (21) and (22) we get

$$
\left|C_{7}^{S M} V_{t b} V_{t s}^{*}+C_{7}^{n e w} V_{t^{\prime} b} V_{t^{\prime} s}^{*}\right|=\sqrt{\frac{\pi f\left(\hat{m}_{c}\right) \kappa\left(\hat{m}_{c}\right)\left|V_{c b}\right|^{2}}{6 \alpha} \frac{\mathcal{B}\left(B \rightarrow X_{s} \gamma\right)}{\mathcal{B}\left(B \rightarrow X_{c} e \bar{\nu}_{e}\right)}} .
$$


The model parameters can be constrained from the measured branching ratios of the $B \rightarrow$ $X_{s} \gamma$ decay and $\mathcal{B}\left(B \rightarrow X_{c} e \bar{\nu}_{e}\right)=10.4 \%$

$$
\mathcal{B}\left(B \rightarrow X_{s} \gamma\right)= \begin{cases}\left(3.21 \pm 0.43 \pm 0.27_{-0.10}^{+0.18}\right) \times 10^{-4} & {[17]} \\ (3.36 \pm 0.53 \pm 0.42 \pm 0.54) \times 10^{-4} & {[18]} \\ (3.11 \pm 0.80 \pm 0.72) \times 10^{-4} & {[19]}\end{cases}
$$

In our numerical analysis, we will use the weighted average value $\mathcal{B}\left(B \rightarrow X_{s} \gamma\right)=$ $(3.23 \pm 0.42) \times 10^{-4}[20]$ for the branching ratio of the $B \rightarrow X_{s} \gamma$ decay.

Another constraint to the extended CKM matrix element comes from the unitarity condition, i.e.,

$$
\begin{aligned}
\left|V_{u s}\right|^{2}+\left|V_{c s}\right|^{2}+\left|V_{t s}\right|^{2}+\left|V_{t^{\prime} s}\right|^{2} & =1, \\
\left|V_{u b}\right|^{2}+\left|V_{c b}\right|^{2}+\left|V_{t b}\right|^{2}+\left|V_{t^{\prime} b}\right|^{2} & =1, \\
V_{u b} V_{u s}^{*}+V_{c b} V_{c s}^{*}+V_{t b} V_{t s}^{*}+V_{t^{\prime} b} V_{t^{\prime} s}^{*} & =0 .
\end{aligned}
$$

Since charged-current tree-level decays are well measured experimentally they are not affected by new physics at leading order. Therefore, for the parameters $\left|V_{u s}\right|,\left|V_{c s}\right|,\left|V_{c b}\right|$ and $\left|V_{u b} / V_{c b}\right|$ we will make use of Particle Data Group (PDG) constraints [13]. Using the weighted average for $\mathcal{B}\left(B \rightarrow X_{s} \gamma\right)$ and PDG constraint $0.38 \leq\left|V_{c b}\right| \leq 0.044$, from Eqs. (24) and (25) we get the constraints

$$
\begin{gathered}
0.011 \leq\left|C_{7}^{S M} V_{t b} V_{t s}^{*}+C_{7}^{n e w} V_{t^{\prime} b} V_{t^{\prime} s}^{*}\right| \leq 0.015 \\
0.03753 \leq\left|V_{t b} V_{t s}^{*}+V_{t^{\prime} b} V_{t^{\prime} s}^{*}\right| \leq 0.043976 \\
0 \leq\left|V_{t s}\right|^{2}+\left|V_{t^{\prime} s}\right|^{2} \leq 0.00492 \\
0.998 \leq\left|V_{t b}\right|^{2}+\left|V_{t^{\prime} b}\right|^{2} \leq 0.9985
\end{gathered}
$$

\section{Numerical analysis}

In this section we will study the constraints to $\left|V_{t^{\prime} b} V_{t^{\prime} s}^{*}\right|$ coming from the measured branching ratios of the $B \rightarrow K \ell^{+} \ell^{-}$and $B \rightarrow K^{*} \ell^{+} \ell^{-}$decays and $B_{s}^{0}-\bar{B}_{s}^{0}$ mixing, as well as using the results in Eqs. (26)-(29). The main input parameters involved in calculation of the branching ratios of the $B \rightarrow K \ell^{+} \ell^{-}$and $B \rightarrow K^{*} \ell^{+} \ell^{-}$decays are the form factors, whose values we take from light cone QCD sum rule [21]-[23], where the form factors are expressed in terms of three parameters as

$$
F(s)=\frac{F(0)}{1-a_{F} s+b_{F} s^{2}},
$$

where the values of parameters $F(0), a_{F}$ and $b_{F}$ for the $B \rightarrow K$ and $B \rightarrow K^{*}$ decay are listed in Table 1.

The values of the other input parameters which we use in our numerical calculations are: $m_{b}=4.8 \mathrm{GeV}, m_{c}=1.35 \mathrm{GeV}, m_{B_{s}}=5.369 \mathrm{GeV}, \tau_{B_{s}}=1.64 \times 10^{-12} \mathrm{~s}$ and 


\begin{tabular}{|l|ccc|}
\hline & $F(0)$ & $a_{F}$ & $b_{F}$ \\
\hline$f_{+}^{B \rightarrow K}$ & 0.35 & 1.37 & 0.35 \\
$f_{0}^{B \rightarrow K}$ & 0.35 & 0.40 & 0.41 \\
$A_{1}^{B \rightarrow K^{*}}$ & 0.337 & 0.60 & -0.023 \\
$A_{2}^{B \rightarrow K^{*}}$ & 0.283 & 1.18 & 0.281 \\
$A_{0}^{B \rightarrow K^{*}}$ & 0.470 & 1.55 & 0.68 \\
$V^{B \rightarrow K^{*}}$ & 0.458 & 1.55 & 0.575 \\
$T_{1}^{B \rightarrow K^{*}}$ & 0.379 & 1.59 & 0.615 \\
$T_{2}^{B \rightarrow K^{*}}$ & 0.379 & 0.49 & -0.241 \\
$T_{3}^{B \rightarrow K^{*}}$ & 0.261 & 1.20 & 0.098 \\
\hline
\end{tabular}

Table 1: $B$ meson decay form factors in a three-parameter fit, where the radiative corrections to the leading twist contribution and $\mathrm{SU}(3)$ breaking effects are taken into account $($ see $[22,23])$.

$B_{B_{s}} f_{B_{s}}^{2}=(0.26 \mathrm{GeV})^{2}$. The experimental lower bound of the mass difference is $\Delta m_{B_{s}} \geq$ $14.9 \mathrm{ps}^{-1}$. For the values of the Wilson coefficients $C_{7}^{S M}, C_{9}^{S M}$ and $C_{10}^{S M}$ we have used their next-to-leading logarithmic result: $C_{7}^{S M}=-0.308, C_{9}^{S M}=4.154$ and $C_{10}^{S M}=-4.261$. It should be noted that the decays $B \rightarrow K \ell^{+} \ell^{-}$and $B \rightarrow K^{*} \ell^{+} \ell^{-}$receive long distance contribution coming from $\bar{c} c$ intermediate states. In the present work we neglect such long distance effects. The strong dependence on $m_{t^{\prime}}$ (see for example [4]) makes the electroweak penguins a good place looking for the existence of fourth generation. Contributions of fourth generation to $B \rightarrow K\left(K^{*}\right) \ell^{+} \ell^{-}$decays have already been studied (see second references in [4] and [6]). The present investigation differs from the above-mentioned works in two aspects:

- we use the experimentally measured results on branching ratio,

- we consider $V_{t b} V_{t s}^{*}$ and $V_{t^{\prime} b} V_{t^{\prime} s}^{*}$ as two independent complex parameters, which were taken to be real in [4] and [6].

The complex parameters $V_{t b} V_{t s}^{*}$ and $V_{t^{\prime} b} V_{t^{\prime} s}^{*}$ are constrained by the unitarity conditions (see Eqs. (27)-(29)), the measured branching ratios $B \rightarrow X_{s} \gamma$ (see Eq. (26)) and $B \rightarrow$ $K\left(K^{*}\right) \ell^{+} \ell^{-}$decays (see Eqs. (15), (16)), which depend on $m_{t^{\prime}}$. For each value of $m_{t^{\prime}}$ there exists an allowed region in the $\left|V_{t b} V_{t s}^{*}\right|-\left|V_{t^{\prime} b} V_{t^{\prime} s}^{*}\right|$ plane.

Since there exists no analytical solution of Eqs. (26)-(29), we will solve these equations numerically assuming that $V_{t b} V_{t s}^{*}$ and $V_{t^{\prime} b} V_{t^{\prime} s}^{*}$ are complex. For sufficiently large number of randomly chosen complex parameters $V_{t^{\prime} b} V_{t^{\prime} s}^{*}$ and $V_{t b} V_{t s}^{*}$, the selected values would range over the whole solution space. In Figs. (1)-(3) we present the allowed region for $V_{t b} V_{t s}^{*}$ and $V_{t^{\prime} b} V_{t^{\prime} s}^{*}$ at $m_{t^{\prime}}=200 \mathrm{GeV}, m_{t^{\prime}}=300 \mathrm{GeV}$ and $m_{t^{\prime}}=400 \mathrm{GeV}$, respectively. In obtaining this solution region we have used Eqs. (26)-(29) and Eq. (15). From these figures we see 
that, for $\left|V_{t^{\prime} b} V_{t^{\prime} s}^{*}\right|=0,\left|V_{t b} V_{t s}^{*}\right|$ takes on values that is close to the SM prediction and is mainly distributed around $\sim 0.04$. Moreover, when $\left|V_{t^{\prime} b} V_{t^{\prime} s}^{*}\right|$ increases, the allowed region of $\left|V_{t b} V_{t s}^{*}\right|$ becomes wider with the center being fixed around 0.04 , and the values of $\left|V_{t^{\prime} b} V_{t^{\prime} s}^{*}\right|$ are mainly distributed around 0.01 . With increasing values of $m_{t^{\prime}}$, the allowed region for $\left|V_{t^{\prime} b} V_{t^{\prime} s}^{*}\right|$ becomes narrower. This behavior can be explained as follows. Wilson coefficients are strongly dependent on $m_{t^{\prime}}$ and in order to remain in the experimentally allowed region, the element $\left|V_{t^{\prime} b} V_{t^{\prime} s}^{*}\right|$ of CKM matrix must decrease, since branching ratio contains factors like $\left|C_{i} V_{t b} V_{t s}^{*}\right|$. Similar behavior is observed for the $B \rightarrow K^{*} \ell^{+} \ell^{-}$decay (see Figs. (4)-(6)).

In Figs. (7) and (8) we present the dependence of $\Delta m_{B_{s}}$ on $\left|V_{t b} V_{t s}^{*}\right|$ and $\left|V_{t^{\prime} b} V_{t^{\prime} s}^{*}\right|$, taking into account the lower experimental bound for $\Delta m_{B_{s}}$, at two different values of $m_{t^{\prime}}$. It follows from both figures that the main distribution is in the range $0.36 \leq\left|V_{t b} V_{t s}^{*}\right| \leq 0.044$ and $0 \leq\left|V_{t^{\prime} b} V_{t^{\prime} s}^{*}\right| \leq 0.01$. With increasing values of $m_{t^{\prime}}$, obviously, $\Delta m_{B_{s}}$ also increases.

Finally we would like to note that restrictions to $\left|V_{t^{\prime} d} V_{t^{\prime} b}^{*}\right|$ matrix element can be obtained by an investigation of the rare decays induced through $b \rightarrow d$ transition. Further analysis of the decays induced by $b \rightarrow s(d)$ transition is more promising in studying new sources for $\mathrm{CP}$ violation, since $4 \times 4 \mathrm{CKM}$ matrix predicts the existence of three $\mathrm{CP}$ violating phases. We will discuss this issue elsewhere in future.

In conclusion, we have studied the effect of the fourth generation quark to the rare decays induced by FCNC $b \rightarrow s$ transition. Using the experimental result for the branching ratios of the $B \rightarrow X_{s} \gamma, B \rightarrow K\left(K^{*}\right) \ell^{+} \ell^{-}$decays and the unitarity condition for the $4 \times 4$ CKM matrix, we have determined the allowed parameter space for $\left|V_{t b} V_{t s}^{*}\right|$ and $\left|V_{t^{\prime} b} V_{t^{\prime} s}^{*}\right|$ in their dependence on $m_{t^{\prime}}$. 


\section{References}

[1] J. I. Silva-Marcos, JHEP 0212 (2002) 036.

[2] H. J. He, N. Polonsky and S. Su, Phys. Rev. D64 (2001) 053004.

[3] V. A. Novikov, L. B. Okun, A. N. Rozanov, and M. I. Vysotsky, Phys. Lett. B529 (2002) 111.

[4] W. S. Hou, A. Soni and H. Steger, Phys. Lett. B192 (1987) 441;

W. S. Hou, R. S. Willey and A. Soni, Phys. Rev. Lett. 58 (1987) 1608; Erratum-ibid 60 (1987) 2337.

[5] A. Arhrib and W. S. Hou, Eur. Phys. J. C27 (2003) 555.

[6] T. M. Aliev, D. A. Demir, N. K. Pak, Phys. Lett. B389 (1996) 83;

T. M. Aliev, A. Özpineci, M. Savc1, Nucl. Phys. B585, (2000) 275;

T. M. Aliev, A. Özpineci, M. Savc1, Eur. Phys. J. C27 (2003) 405.

[7] T. Hattori, T. Hasuike and S. Wakaizumi, Phys. Rev. D60 (1999) 113008.

[8] C. S. Huang, W. J. Huo and Y. L. Wu, Mod. Phys. Lett. A14 (1999) 2453; Phys. Rev. D64 (2001) 016009; W. J. Huo, Eur. J. Phys. C24 (2002) 275.

[9] M. S. Alam, et. al, CLEO Collaboration, Phys. Rev. Lett. 74 (1995) 2885.

[10] K. Abe at. al, Belle Collaboration, Phys. Rev. Lett. 88 (2002) 021801.

[11] B. Aubert et. al, BaBar Collaboration, Phys. Rev. Lett. 88 (2002) 241801.

[12] B. Aubert et. al, BaBar Collaboration, prep. hep-ex/0207082 (2002).

[13] K. Hagiwara et. al, Particle Data Group, Phys. Rev. D66 (2002) 010001.

[14] G. Buchalla and A. J. Buras, Nucl. Phys. B400 (1993) 225.

[15] M. Misiak, Nucl. Phys. B398 (1993) 23; Erratum-ibid. B439 (1995) 461.

[16] A. J. Buras, prep. hep-ph/9806471 (1998).

[17] S. Chen et. al, CLEO Collaboration, Phys. Rev. Lett. 87 (2001) 251807.

[18] H. Tajima, BELLE Collaboration, Int. J. Mod. Phys. A17 (2002) 2967.

[19] R. Barate et. al, ALEPH Collaboration, Phys. Lett. B429 (1998) 169.

[20] P. Gambino, M. Misiak, Nucl. Phys. B611 (2001) 338.

[21] T. M. Aliev, A. Özpineci, M. Savcı, Phys. Rev. D55 (1997) 7059.

[22] P. Ball, V. M. Braun, Phys. Rev. D58 (1998) 094016.

[23] P. Ball, prep. hep-ph/9803501 (1998). 


\section{Figure captions}

Fig. (1) Three-dimensional plot of the branching ratio for the $B \rightarrow K \ell^{+} \ell^{-}$decay, with respect to the allowed parameter space of $V_{t b} V_{t s}^{*}$ and $V_{t^{\prime} b} V_{t^{\prime} s}^{*}$, at $m_{t^{\prime}}=200 \mathrm{GeV}$.

Fig. (2) The same as in Fig. (1), but at $m_{t^{\prime}}=300 \mathrm{GeV}$.

Fig. (3) The same as in Fig. (1), but at $m_{t^{\prime}}=400 \mathrm{GeV}$.

Fig. (4) The same as in Fig. (1), but for the $B \rightarrow K^{*} \ell^{+} \ell^{-}$decay.

Fig. (5) The same as in Fig. (4), but at $m_{t^{\prime}}=300 \mathrm{GeV}$

Fig. (6) The same as in Fig. (4), but at $m_{t^{\prime}}=400 \mathrm{GeV}$.

Fig. (7) Three-dimensional plot of the mass difference $\Delta m_{B_{s}}$ of the $B_{s}^{0}-\bar{B}_{s}^{0}$ system, with respect to the allowed parameter space of $V_{t b} V_{t s}^{*}$ and $V_{t^{\prime} b} V_{t^{\prime} s}^{*}$, at $m_{t^{\prime}}=300 \mathrm{GeV}$.

Fig. (8) The same as in Fig. (7), but at $m_{t^{\prime}}=700 \mathrm{GeV}$. 


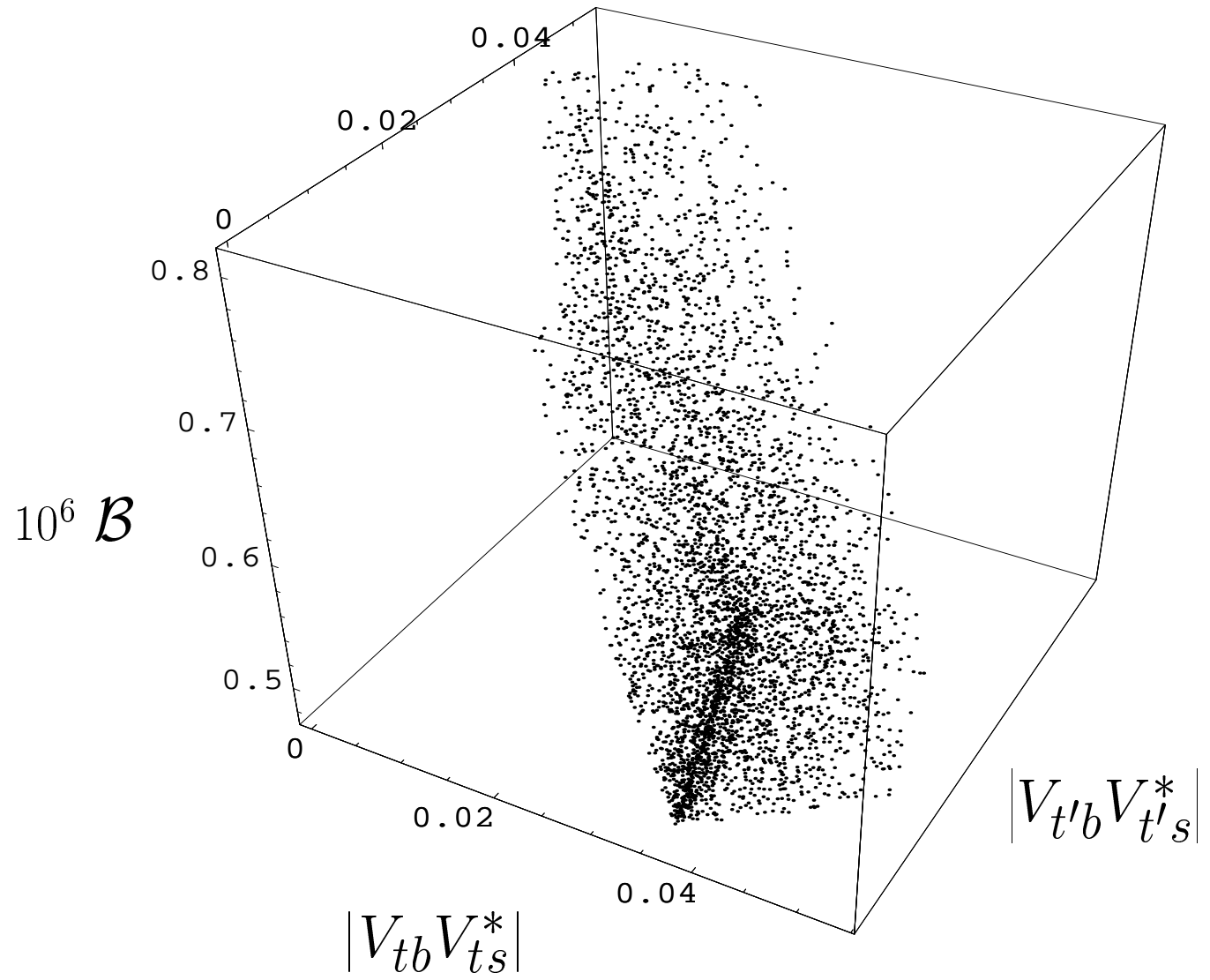

Figure 1: 


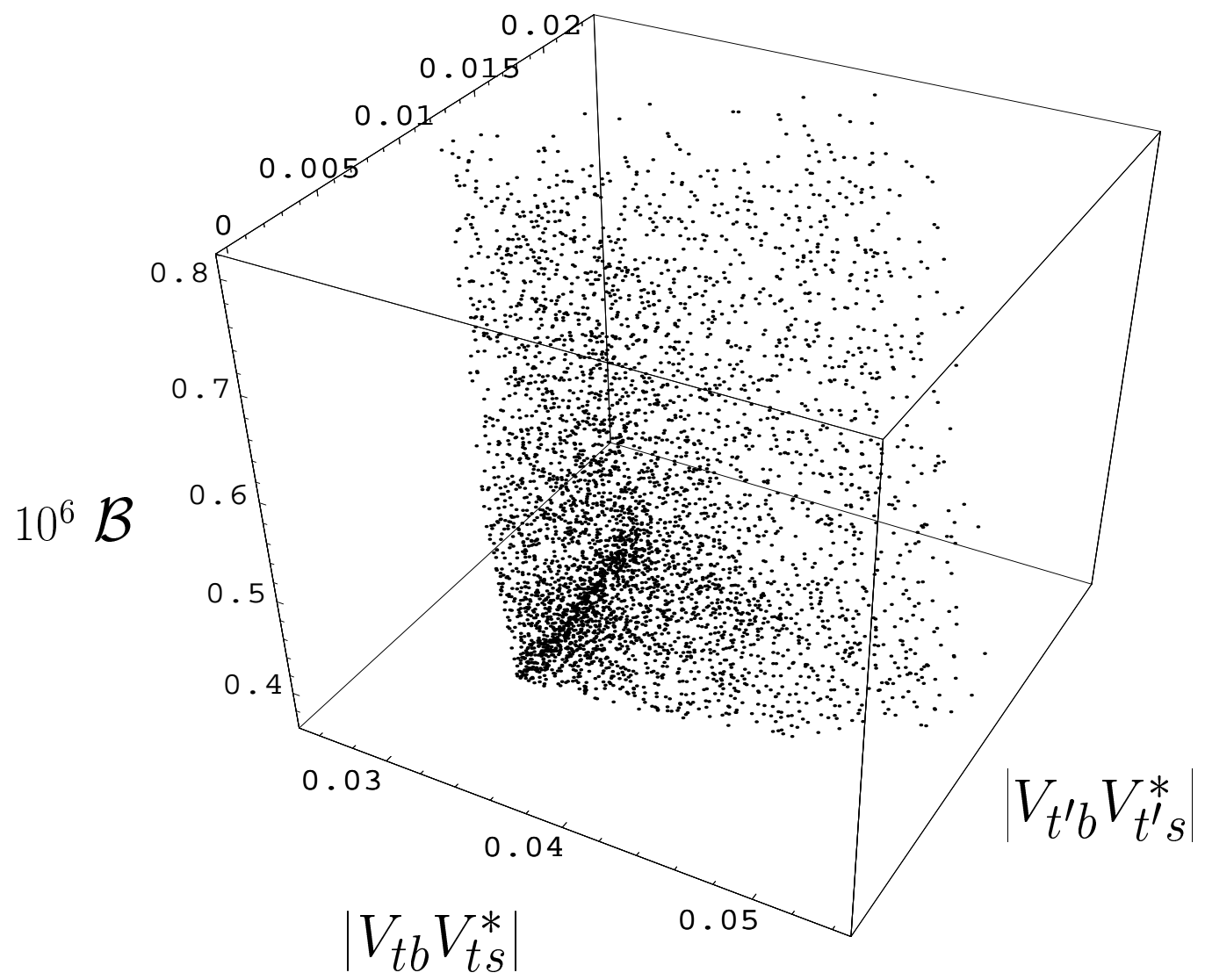

Figure 2: 


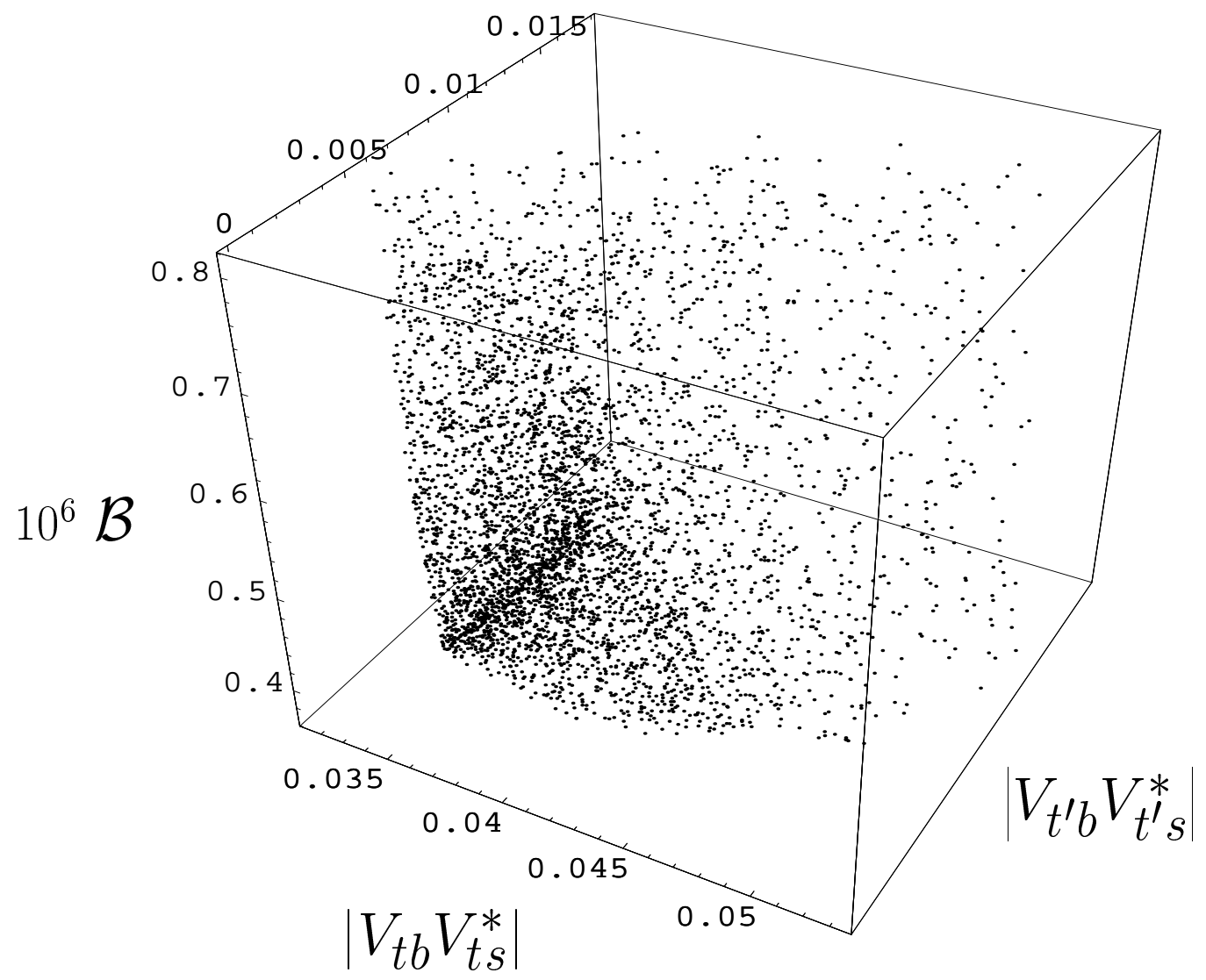

Figure 3: 


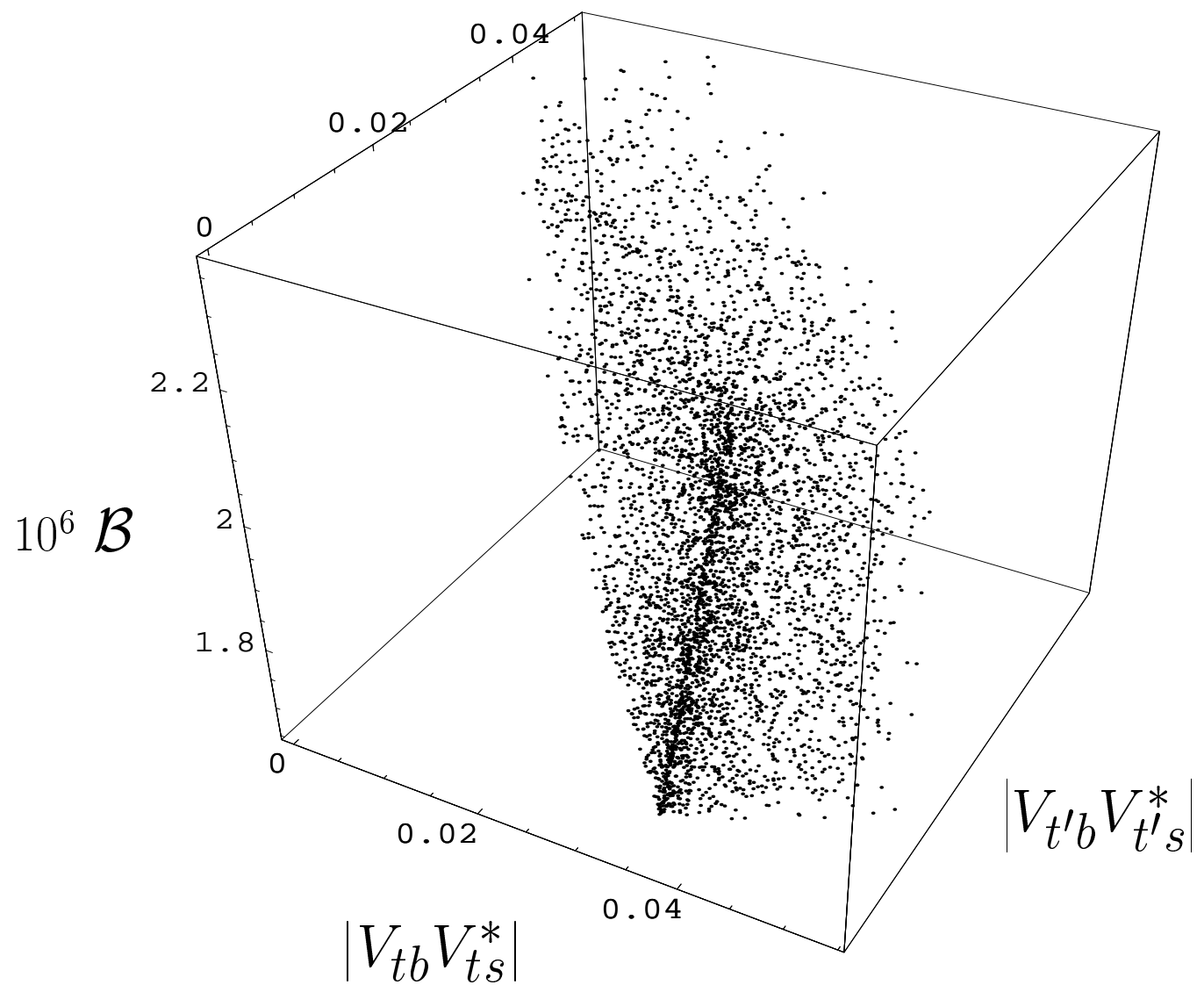

Figure 4: 


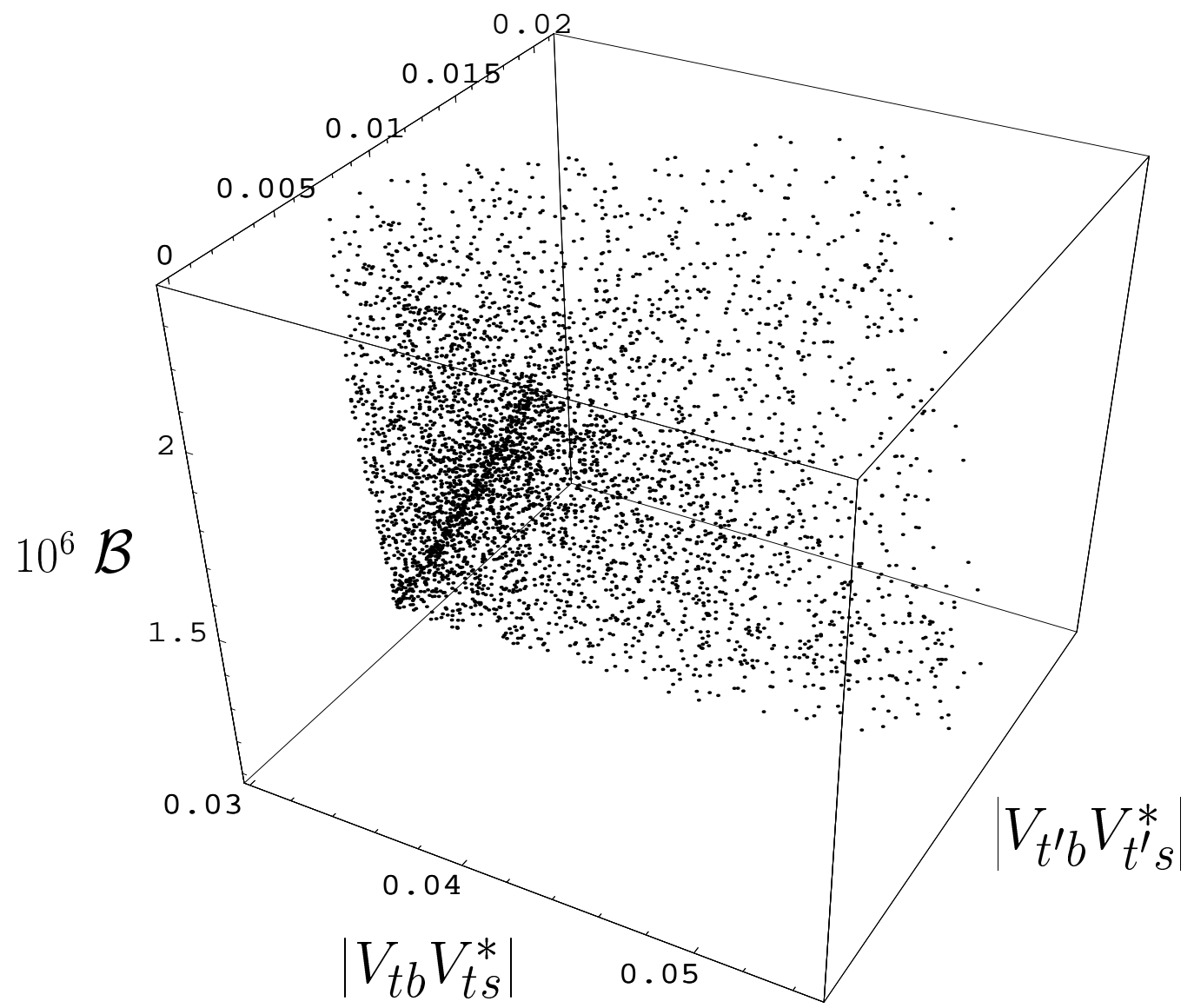

Figure 5: 


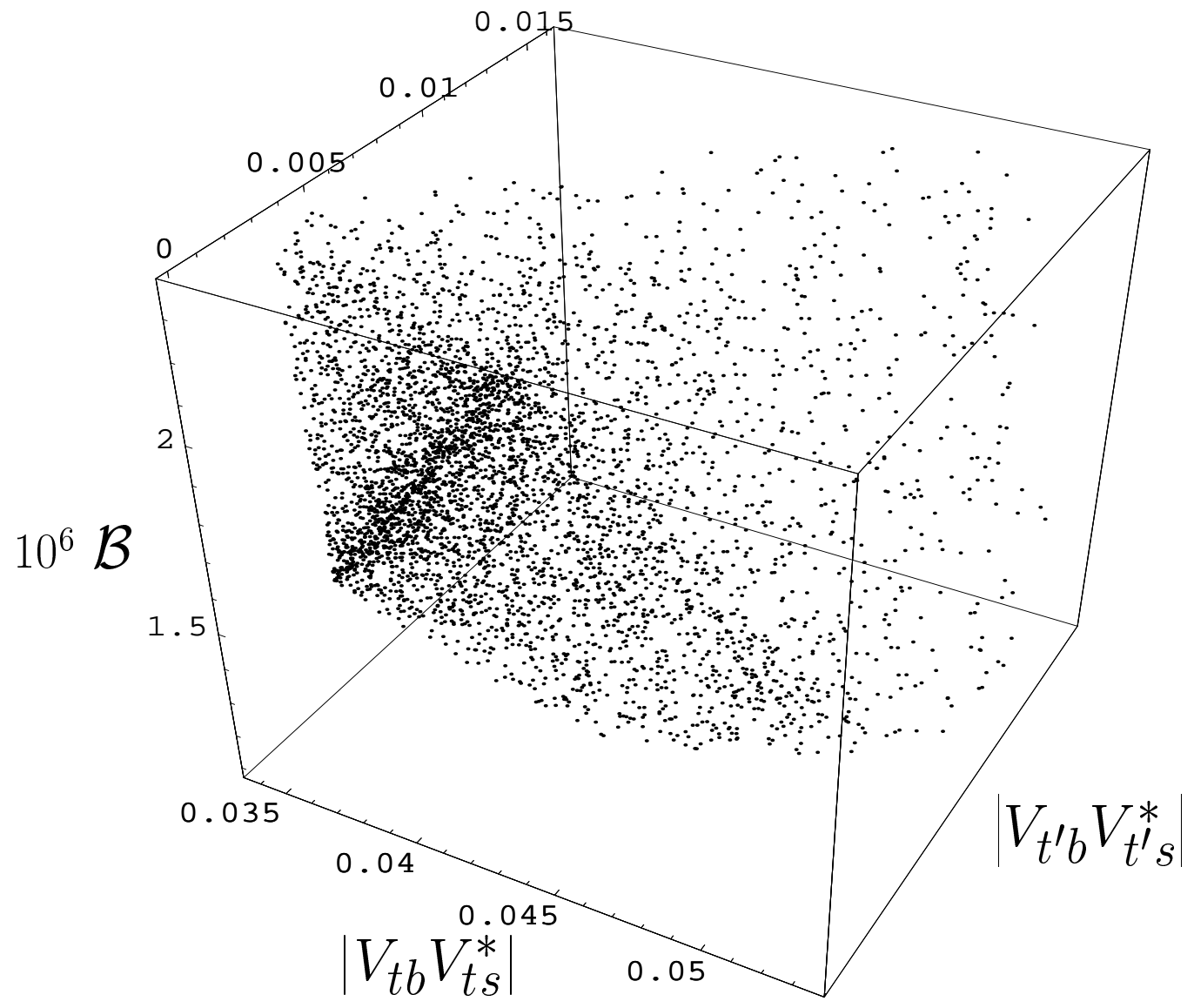

Figure 6: 


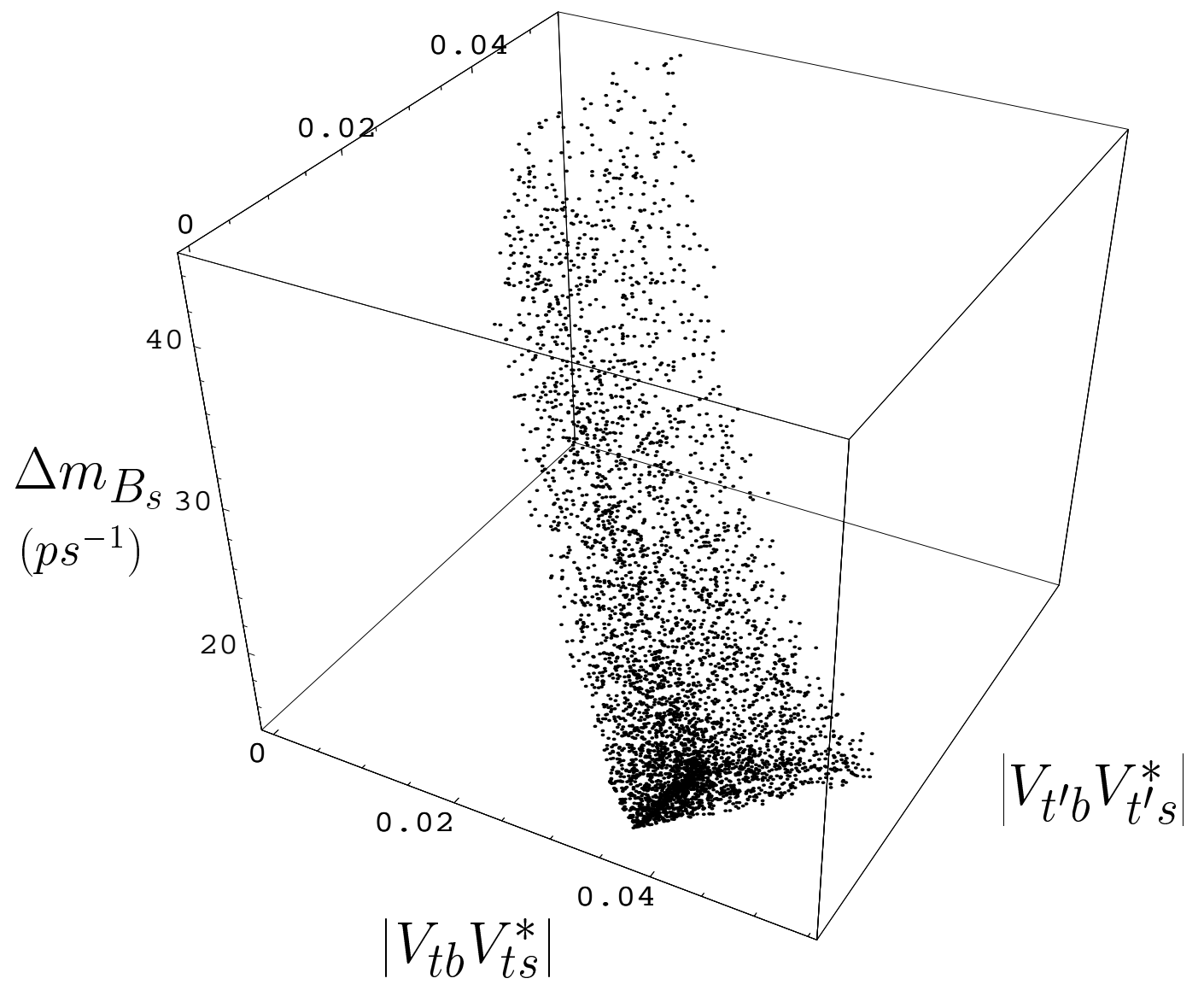

Figure 7: 


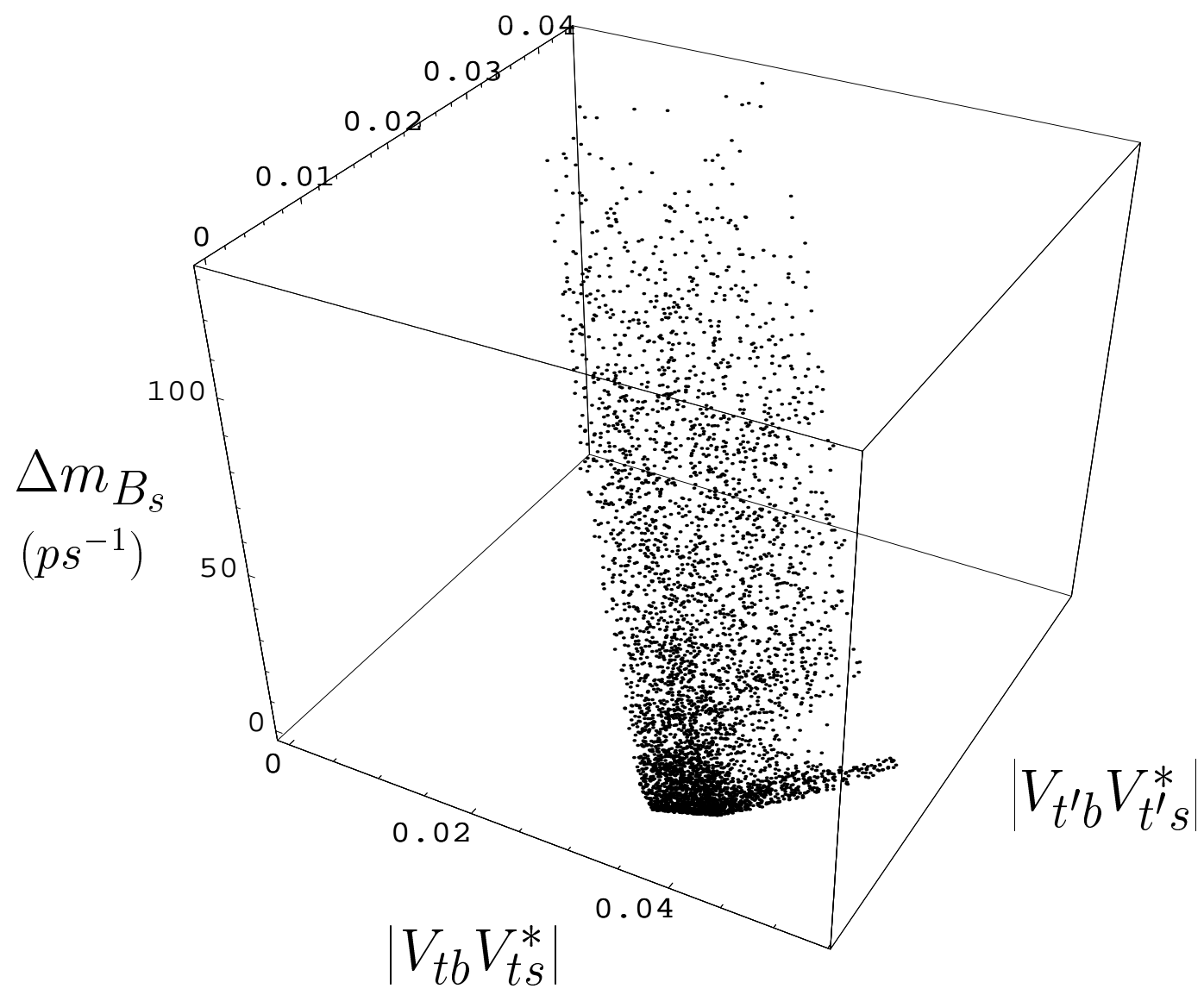

Figure 8: 